\title{
MICRO-84
}

\section{Electron microscopy 50 years on}

from Colin J. Humphreys

IN 1934, Nature published the first electron micrographs of biological material. Fifty years later, the Royal Microscopical Society, in association with Nature, organized MICRO-84, an international microscopy conference and exhibition held in London from 9 to 13 July 1984. The conference was attended by about 800 scientists from all over the world.

One of the highlights of the meeting was a description by Albert Crewe (University of Chicago) of a revolutionary $0.6 \AA$ point resolution scanning transmission electron microscope he has recently started to build. This represents a dramatic advance in resolution over existing electron microscopes, the best of which have a point resolution of about $1.7 \AA$. Modern electron microscopes have computer control added on, as it were. Crewe proposes to reverse this procedure and build his microscope around a computer system large enough to operate and control the microscope, and process and store the images. His computer system, comprising four computers with 3.6 Giga bytes of storage, has been donated by IBM, which demonstrates the importance that major microelectronics companies attach to high-resolution electron microscopy. If successfully built, Crewe's microscope should have major applications in biology, chemistry, materials science and physics.

Many microelectronic circuits are now so small that they cannot be resolved using an optical microscope, and an electron microscope is necessary simply to see them. D. Cherns (University of Bristol) and W.M. Stobbs and D.J. Smith (University of Cambridge) demonstrated how highresolution electron microscopy can be used to determine the positions of atoms lying in and near the interfaces of semiconductor devices, so that their electrical properties can be understood.

In recent years, not only the resolution but also the analytical capabilities of electron microscopes have dramatically improved. It is now possible to analyse chemically very small regions of materials using an electron beam less than $10 \AA$ in diameter, by detecting the signals (for example, characteristic $X$ rays) generated when the incident electron beam is inelastically scattered by the specimen. Moreover, it is possible to measure the energy spectrum of the transmitted electrons by a technique called electron energy loss spectroscopy, which is sensitive to all elements and is increasingly being used for the analysis of both biological and non-biological materials.

If the fine structure of an electron energy spectrum is studied, a technique known as electron loss near edge structure (ELNES), results can be obtained equivalent to those achieved on a synchrotron source, but for any atomic number element and with far higher spatial resolution (C. Colliex, University of Paris). ELNES tells us not only what atoms are present in a sample, but also their environment. Mick Brown (University of Cambridge) described some novel applications of electron energy loss spectroscopy which exploit the very high spatial resolution available, for example, the measurement of energy band gaps in insulators.

Topics covered in the wide-ranging programme included microsurgery, for- ensic science, archaeology and art. Among the relatively new fields, C.F. Quate (Stanford University) gave an outstanding review of the advances in acoustic microscopy from a resolution of $10 \mu \mathrm{m} 10$ years ago to one of $300 \AA$, using helium, today, and G. Schmahl (University of Gottingen) described the latest results from his $\mathbf{5 0 0}$ $\AA$ resolution X-ray microscope, which is probably the best in the world. X-ray microscopy offers the prospect of studying living biological material with a resolution far better than that of optical microscopy, and with much less damage than is produced in an electron microscope. To be really useful, however, its resolution will have to be improved still further. Perhaps this will be achieved by the next meeting of MICRO, in 2 years time.

Colin J. Humphreys is in the Department of Metallurgy and Science of Materials, University of Oxford, Parks Road, Oxford OXI $3 P H$.

\section{Vaccine development}

\section{Recombinant vaccinia for prevention of hepatitis B}

from John Beale

THE idea of using vaccinia virus as a means of carrying other immunogens for the purpose of vaccination has seemed very attractive since Paoletti and Moss and their colleagues started to investigate the use of vaccinia recombinants in 1982 (Panicali, D. \& Paoletti, E. Proc. natn. Acad. Sci. U.S.A. 79, 4927; 1982 and Mackett, M., Smith, G.L. \& Moss, B. op. cit. 79, 7415; 1982). The rationale is that the live virus will express a foreign gene inserted into its DNA when it is applied to an animal, which will subsequently produce an immunological response to the foreign gene product. So far, recombinant DNA technology has been used successfully to express various antigens in vaccinia, including hepatitis B virus surface antigen (HBsAg), malaria sporozoite antigen, and influenza and herpes glycoproteins, and the list is growing all the time. On page 67 of this week's Nature, Moss and his colleagues take the hepatitis story a stage closer to a practical vaccine by showing that chimpanzees can be protected against hepatitis by vaccination with live recombinant vaccinia virus.

Last year, Smith, Mackett and Moss reported the construction of a fusion gene between DNA encoding $\mathrm{HBsAg}$ and an early vaccinia promoter (UCLA Symp. molec. cell. Biol., New Ser. 8, 543; 1983). By inserting the fusion product into the thymidine kinase-encoding portion of the vaccinia genome such that thymidine kinase activity was no longer expressed, they could select the new recombinant virus by growth in the presence of 5 -bromodeoxyuridine. The plaques were tested for HBsAg expression by dot-blot hybridization and for $\mathrm{HBsAg}$ gene expression by immunological methods.

What Moss and his colleagues have now done is to vaccinate two chimpanzees with the $\mathrm{HBs} \mathrm{Ag}$-containing recombinant vaccinia virus and one chimpanzee with wild-type normal vaccinia virus, and then challenge them with living hepatitis $B$ virus. They find that treatment with $\mathrm{HBsAg}$ vaccinia hybrids produces smaller lesions and lower antibody titres to vaccinia than does treatment with vaccinia alone. None of the animals produced antibodies to $\mathrm{HBsAg}$ after vaccination. On challenge with hepatitis B virus, however, the chimpanzee given vaccinia alone developed typical signs of hepatitis B virus infection: hepatitis surface antigenaemia, antibodies to the core antigen as well as to the surface antigen of hepatitis B (the production of core antibodies indicates some hepatitis B virus replication had occurred in the primed animal) and biochemical evidence of hepatitis. By contrast, the two $\mathrm{HBs} \mathrm{Ag}$ vaccinia-treated chimpanzees, although producing no antibodies before challenge, were protected against the challenge, as shown by the lack of biochemical evidence of liver damage and of antigenaemia, and the rapid onset of antibodies to the surface antigen. They had thus been immunologically primed by the expression of $\mathrm{HBs} \mathrm{Ag}$ when the recombinant vaccinia was growing in the skin.

These are impressive results that pose in a sharper form the policy implications of this approach. The attraction is that the accepted and proven instrument of smallpox eradication can be adapted to the formidable task of hepatitis B prevention. 
Indeed, as a result of the success of the World Health Organisation (WHO) smallpox eradication campaign, vaccinia has had magical powers conferred on it, not only by the populations that have benefitted; but also by some public health officials.

At the time of the first publication by Smith, Mackett and Moss, two problems were foreseen: immunity to vaccinia and the question of safety. These two issues remain. On any rational consideration of cost versus benefit, vaccinia should be acceptable in Third World countries for the prevention of hepatitis B. Such decisions are not always made rationally, however, and the use of a vaccinia hybrid for the control of hepatitis B in the USA, for example, would probably be more convincing to governments in Third World countries than hours of debate. For western countries, however, there is no incentive to use vaccinia with its known hazards, which caused it to be abandoned for smallpox control around the time that the WHO started its smallpox eradication scheme. Moss et al.'s finding that the lesions in the chimpanzees were less for the recombinant than for the wild vaccinia is encouraging, although more work is clearly needed. The problems of effectiveness and of interference from vaccinia antibodies during booster doses remain to be overcome.

As large amounts of hepatitis B surface antigens become available using recombinant DNA techniques of expression in yeasts and in mammalian cells, there may be no need to use a vaccinia carrier. Instead, it may be possible to combine hepatitis B vaccine with other nonliving childhood vaccines, and to give the combination at birth or soon thereafter.

Priming of the immune response so that immunological memory is induced is one aim of a vaccination programme. Recently, Salk et al. (Ann. clin. Res. 14, 204 ; 1982) have argued forcibly that this would justify consideration of a single dose of killed poliovaccine for poliomyelitis control in tropical areas. The problem is the uncertain duration of memory in the absence of an adequate primary stimulus to produce antibody of both IgM and IgG classes. Further experimental data should soon be available to answer these outstanding questions for poliovaccine.

As to future research into the use of vaccinia as a carrier of immunogens, increased expression of the inserted DNA so that larger quantities of immunogen are produced is an important goal. Certainly, there is sufficient evidence to warrant continued research and the cautious extension of experiments in man. It is, however, much too early to think this promising line of research is a panacea for all the vaccine problems in developing countries.

John Beale is at Wellcome Biotechnology Ltd, Langley Court, Beckenham, Kent BR3 $3 B S$.
Mathematical modelling

\section{The cubic map in theory and practice}

from Robert M. May

FOR many populations of temperate-zone insects, the adults emerge, mate, reproduce and die over a brief interval in the summer. The dynamical behaviour of such a population, with discrete nonoverlapping generations, may be described by a firstorder difference equation of the form $x_{t+1}$ $=f\left(x_{t}\right)$; the population density in generation $t+1, x_{t+1}$, is given as some function, $f$, of the density in generation $t$, $x_{t}$. If this equation were linear $(f=\lambda x)$, the population would simply increase (or decrease if $\lambda<1$ ) in familiar exponential fashion. More commonly, however, the population will tend to increase when at low densities and to crash at high densities, corresponding to some non-linear function or 'map' with a hump (of which the quadratic $f=\lambda x(1-x)$ is the mathematically simplest example). It is now widely appreciated that such non-linear firstorder difference equations with a single hump or critical point can unfold an astonishing richness of dynamical behaviour: as nonlinearities become more severe (specifically, as $\lambda$ increases in the quadratic example), the original stable point gives way to a cascade of perioddoubling bifurcations, successively producing cycles of periods $2,4,8,16, \ldots$, $2^{n}$; eventually an apparently 'chaotic' regime is entered, in which the trajectories generated by this deterministic process can be effectively indistinguishable from random noise $\mathrm{e}^{1,2}$. Many features of this bifurcation phenomenon are reproduced in a self-similar manner, on even finer scales, in successive period doublings, in ways that are generic to essentially all maps with one critical point ${ }^{1-3}$. This subject, which first came to prominence in the early 1970 s in studies of biological populations ${ }^{4,5}$, has grown dramatically as more possible applications are found in the physical and engineering sciences.

What are the properties of maps with two humps? The canonical example of a map with two critical points is the symmetrical cubic $x_{t+1}=\lambda x_{t}+(1-\lambda) x_{t}^{3}$ (which is chosen such that +1 maps to + 1,0 to 0 and -1 to -1 ). A hint that the dynamics of the cubic are likely to be messier than those of the quadratic is provided by a theorem saying that maps with $n$ critical points can have up to $n$ distinct stable orbits for any given set of parameter values $^{6}$. For maps with one hump, this means at most one stable state or attractor; for generically cubic maps, however, there can be two alternative attractors. Several authors have set out how two such alternative stable orbits can arise for the cubic map ${ }^{7-9}$ : when the originally stable point at $x=0$ becomes unstable (for $\lambda>2$ ), it first bifurcates to give a single symmetrical cycle with period 2; as the map becomes more significantly nonlinear $(\lambda>3)$, this cycle does not bifurcate to give a 4 -cycle, but rather splits to give two distinctly different (and unsymmetrical) 2-point cycles. Each of the separate 2-point cycles then unfolds the familiar cascade of period doublings to cycles with periods $4,8,16, \ldots, 2^{n}$ as $\lambda$ continues to increase. These cascades display the self-similar properties emphasized by Feigenbaum ${ }^{3}$ for the quadratic map. For yet larger values of $\lambda$ we enter the apparently 'chaotic' regime, where, again, there are two separate attractors for most values of $\lambda$. A detailed catalogue of all the possible cycles that lurk as a kind of kaleidoscopic fine structure within the apparently chaotic regime is much more complicated than for the quadratic map ${ }^{1,2}$, though an explicit such catalogue has been given for all cycles with periods up to 10, in their order of appearance 9 . Methods for computing the total number of distinct cycles with period $k$ have also been given ${ }^{7}$.

The motive behind the first studies of the cubic map was simply that it represented the next logical step beyond the quadratic ${ }^{7}$. Since then, however, the cubic map has come to people's attention in a variety of practical contexts.

Holmes ${ }^{10}$ studied equations that arise as the simplest description of a buckled beam that is subjected to forced lateral vibrations. This led him naturally to consider the cubic equation given above, as being the simplest dynamical relationship satisfying the symmetry requirements of his problem. Holmes then established that, for a wide

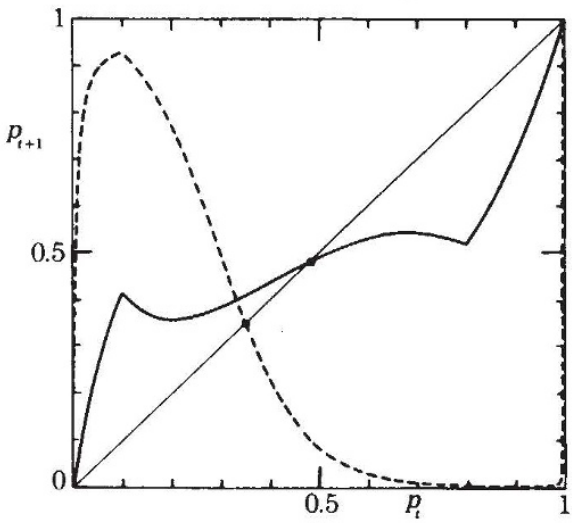

Relationship between the frequency of allele $A$ in successive generations, $p_{\mathrm{t}+1}$ to $p_{\mathrm{t}}$, for a system in which the fitness of host genotypes are determined by associations with pathogens. It is the general propensity to increase at low values and decrease at high values that is the characteristic of the cubic map. (From ref.16.) 